\title{
Significado de la naturaleza desde la cosmovisión del pueblo rama en las comunidades de Indian River y Punta de Águila del territorio rama - kriol
}

\author{
Meaning of nature from the world view of the rama people in the Indian River and Punta de Águila \\ communities from the rama - kriol territory
}

Selvano Ervin Hodgson ${ }^{1}$

\section{Resumen}

La investigación sobre el "Significado de la naturaleza desde la cosmovisión del pueblo rama en las comunidades de Indian River y Punta de Águila del territorio rama - kriol", para el fortalecimiento de la identidad cultural del pueblo rama fue realizada en el 2018, principalmente en las comunidades de Bangkukuk ${ }^{2}$ y Indian River ${ }^{3}$, San Juan de Nicaragua. La investigación, es cualitativa con enfoque etnográfico, ha identificado y documentado los conocimientos de las prácticas de la cultura, ha propuesto estrategias para la incorporación de estos valores culturales en el currículo de Educación Intercultural Bilingüe para el pueblo rama.

Palabra clave: Cosmovisión; identidad cultural; naturaleza; mitología; pueblo rama; historia oral.

\section{Summary}

The research on the Meaning of nature from the world view of the rama people in the Indian River and Punta de Águila communities from the rama - kriol territory, for the strengthening of the cultural identity of the rama people was carried out in 2018 mainly in Bangkukuk and Indian River communities, San Juan de Nicaragua. The research has identified and documented the knowledge of the practices of culture, it has proposed strategies for the incorporation of these cultural values in the Bilingual Intercultural Education curriculum for the rama people.

Keywords: World view; Cultural Identity; Nature; Mythology; rama People; Oral History.

\footnotetext{
2 Bangkukuk es una de las 9 comunidades del Territorio rama kriol. Actualmente habitan la mayor cantidad de hablantes de la lengua rama

3 Lugar donde habitaba los primeros pobladores indígena rama antes de la llegada a la isla de Rama Cay y Punta Gorda
}

Recibido: 14/02/2020 Aprobado: 29/05/2020 


\section{Introducción}

Esta investigación del pueblo rama "Significado de la naturaleza desde la cosmovisión del pueblo rama en las comunidades de Indian River y Punta de Águila del territorio rama - kriol" se contextualiza desde el enfoque del pueblo rama ejecutado desde la universidad URACCAN, y el Instituto IPILC, recinto Bluefields, Región Autónoma de la Costa Caribe Sur (RACCS) en las comunidades de Punta de Águila y Indian River, San Juan de Nicaragua, socializando contenidos sobre los conocimientos ancestrales, culturales e históricos basados en la práctica de epistemología y el paradigma indígena, contribuyendo al desarrollo que fortalece la identidad cultural del pueblo rama, desde la convivencia epistemológica, y los valores ancestrales.

Los indígenas rama son un pueblo que vive en el bosque tropical, en las márgenes de los ríos y en las áreas costeras del sureste de Nicaragua. Según Gerald Riverstone (2008), el pueblo rama identifica que su territorio ancestral se extiende hacia el sur, desde el río Escondido hasta el río San Juan, a lo largo de la actual frontera entre Nicaragua y Costa Rica.

El proyecto de investigación sobre los conocimientos ancestrales del pueblo indígena rama, contribuye con nuevo estudio etnográfico; asimismo fortalece el proceso de la revitalización cultural y lingüística que han desarrollado desde El Instituto de promoción e investigación lingüística y Rescate Cultural IPILC (URACCAN-IPILC) en la promoción e implementación basada en estudios de los pueblos indígenas en la Costa Caribe de Nicaragua, se proporciona informaciones relevantes en la que, favorece para un promover el enfoque intercultural de género y desde la perspectiva de una educación intercultural para la comunidades étnicas e indígenas.

Todos estos conocimientos ancestrales del pueblo rama, benefician a la comunidad indígena rama en el territorio y a la comunidad de aprendizaje como elementos que servirá para un próximo estudio sociológico, retomando eso elementos culturales desde la perspectiva intercultural desde la cosmovisión indígena.

Los esfuerzos que se han hecho para promover y proteger la diversidad cultural e histórica que el mundo enfrenta en un importante reto: en primer lugar, defender la capacidad creativa de cada una de las culturas de los pueblos indígenas y afrodescendientes $y$, en segundo lugar, garantizar la convivencia pacífica entre pueblos que poseen diversas culturas y el respeto de armonía con la madre naturaleza. 


\section{Revisión literaria}

\section{Fundamentos teórica de las sabidurías y conocimientos}

\section{Cultura y conocimientos ancestrales}

La cultura crea sentimientos de identidad común y de pertenencia, los cuales son elementos claves para la cohesión social. Esta cohesión social. Esto es fundamental para el impulso del proceso de consolidación y profundización del régimen autonómico del cual gozan ambas Regiones. Y, siendo que la cultura es entendida por los pueblos indígenas, comunidades afrodescendientes y mestizos costeños. Las culturas de los pueblos indígenas están basadas en sus conocimientos tradicionales, su visión del mundo y su espiritualidad. Éstos no son estáticos, al contrario, son dinámicos: producen innovaciones desde dentro, abarcan todos los aspectos de la vida comunitaria, se vinculan al manejo del entorno natural, son acumulativos y representan generaciones de experiencias, observación cuidadosa y experimentación constante, pero también usan y adaptan conocimientos externos (Cuenin, F. 2009)

Mario Rizo (2002) afirma que, la cultura el conjunto de conocimientos, saberes, principios, técnicas, representaciones simbólicas que comparten los integrantes de un determinado pueblo. Sus costumbres, ordenamiento y creencias los distingue de los demás pueblos.

La cultura de un grupo abarca la forma de vida específica y distinta de ese grupo; es decir los significados, valores e ideas que se manifiestan entre las instituciones, las relaciones sociales, el uso de los objetos y la vida material. Una cultura contiene los mapas de significados mediante los cuales sus miembros entienden la vida (Heckt, 2004)

Los derechos de los pueblos indígenas tienen una relación muy cercana a los derechos culturales, aunque tiene carácter, objetivos y alcances particulares. Estos derechos son esencialmente colectivos; por otro lado, si bien el aspecto es importante, no es la única dimensión de los derechos específicos de los pueblos (Stavenhagen, 2002: 43)

Los conocimientos tradicionales del pueblo indígena rama se manifiestan en forma reservada y responden a sus necesidades y aspiraciones; las creencias, valores, y prácticas, todo este conjunto de conocimientos, creencias y saberes de las comunidades en el territorio rama en la vida práctica son compartidos y están orientados hacia la conservación para educar a la nueva generación.

Quiero decir que todas las culturas están dotadas de valores específicos, aptos para desarrollarse, siempre que se parta del principio de la igualdad de las culturas. Esto supone la igualdad de los pueblos. En cuanto el proceso de revitalización de 
los conocimientos tradicionales esto servirá para el fortalecimiento de la identidad cultural y lingüística

Según Roncal (2006), el conocimiento indígena es un conjunto integrado de saberes $\mathrm{y}$ vivencias de las culturas fundamentadas en nuestras experiencias, y su proceso de interacción permanente hombre-naturaleza y la divinidad; las sabidurías ancestrales y el conocimiento colectivo tienen un vínculo imprescindible con las tierras y territorios, y la existencia como pueblos indígenas; los sistemas de propiedad intelectual no son una institución que deba contemplar disposiciones acerca de los saberes ancestrales y conocimientos colectivos de los pueblos indígenas.

Los conocimientos tradicionales, las innovaciones y la creatividad, incluido la "tradición", han recibido una atención creciente en numerosas esferas de política, que van desde la alimentación y la agricultura al desarrollo económico y comercial pasando por el medio ambiente, la salud, los derechos humanos y las políticas culturales. En varias esferas políticas se está examinando la función de los derechos de propiedad intelectual para proteger los conocimientos tradicionales de un pueblo dada la práctica de existencia.

\section{Espiritualidad y cosmovisión}

La espiritualidad, conocimientos y pensamientos de los pueblos indígenas de Abya Yala son las bases sobre las cuales se definen sus formas y proyectos de vida. Las características intrínsecas de los pueblos indígenas, especialmente los derechos a sus tierras, territorios y recursos, derivan de sus estructuras políticas, económicas y sociales, sus culturas, tradiciones espirituales, su historia y su concepción de la vida. A través de este módulo los participantes contarán con un espacio de recreación cultural que les permitirá profundizar en los elementos que han facilitado la resistencia y transformación de los pueblos indígenas en actores políticos importantes en cada uno de los países de la región (Cunningham, M. 2008).

Los pueblos indígenas contribuyen a la riqueza y diversidad de las civilizaciones y culturas. Diversos instrumentos internacionales y nacionales de protección de los derechos humanos reconocen que el respeto de los conocimientos, las culturas y las prácticas tradicionales indígenas contribuyen al desarrollo sostenible y equitativo, así como al ordenamiento del medio ambiente. Los estudios sobre esos conocimientos, sin embargo, se han hecho generalmente desde la posición de la ciencia occidental, que ha puesto en situación de subordinación los conocimientos indígenas.

Para los pueblos indígenas la espiritualidad es energía, esencia y acción; el espíritu está dentro de la materia. El espíritu es la esencia que da vida a la materia y aquí la relación intrínseca con el cosmos, donde se conjugan las fuerzas energéticas de los 
seres que habitamos esta tierra (sentimientos, pensamientos, acciones, etc.). Así mismo, la visión cósmica de la vida es estar conectado con el entorno, dado que todo lo que hay en el entorno tiene vida, por lo que adquiere un valor sagrado (Cox, A, 2016).

La espiritualidad nace de esta visión y concepción en la que todos los seres que hay en la Madre Naturaleza tienen vida y se interrelacionan. La espiritualidad está ligada al sentido comunitario, donde los seres se interrelacionan y se complementan en su existencia. El propósito fundamental de la espiritualidad es la búsqueda del equilibrio y la armonía con nosotros mismos y con los demás (cosmos). La espiritualidad tiene varias manifestaciones y medios para lograr el equilibrio, entre ellas: el fuego sagrado, las ceremonias, lugares sagrados, cerros, montañas, ríos, lagos, cantos y ofrendas.

La cosmovisión es la forma de concebir, explicar y vivir la vida desde los pueblos; fundamenta los sistemas de vida, sistemas jurídicos y de organización político-social, así como el conjunto de valores, principios, creencias, normas y todas las formas de manifestaciones y expresiones propias de los pueblos.

\section{Simbología ancestral}

Cunningham, M. (2018) plantea que, los conocimientos son el resultado de procesos acumulativos y cooperativos de un pueblo, ya que se van produciendo a lo largo de toda la existencia de un pueblo o grupo social. El conocimiento de un pueblo es por lo tanto, un patrimonio colectivo. Los conocimientos surgen de la existencia práctica. Es decir, de la respuesta que el pueblo da a sus necesidades y problemas. No se puede inventar una solución si no existe un problema y nadie puede formular un problema si no ha tenido una experiencia previa que implique ese problema. Los temas de interés, el contenido del conocimiento, su forma de expresión en palabras, cantos, bailes, cuentos, poemas o símbolos explicativos, la forma de conservar, compartir, utilizar y transmitir esos conocimientos, así como sus productos y objetos materiales estarán identificados con la existencia concreta de cada pueblo y con sus objetivos colectivos.

Los conocimientos de los pueblos indígenas se transmiten a través de narraciones, cantos épicos, música, ceremonias; también sembrando, cosechando, venerando en sitios sagrados, celebrando ritos anuales. Los conocimientos son fundamentalmente el resultado de un proceso acumulativo y cooperativo que se va produciendo a lo largo de toda la existencia de un grupo social. El conjunto acumulado de conocimientos es un patrimonio colectivo.

\section{Medicina tradicional}

Es el médico de mayor rango dado que es enviado especial con mucho poder curativo que puede predecir cualquier fenómeno sobrenatural en sus actividades; además, realizan muchos prodigios poco vistos en otros médicos, entre sus misiones 
está la de contener catástrofes, evitar plagas de los cultivos, limpiar comunidades de malos espíritus cuando realizan las curaciones y en sus momentos ritualísticos exigen muchas observaciones a los comunitarios como no cortar árboles, no prender fuego cerca del lugar preparado, no destazar animales, es decir, no puede haber infracciones, estos médicos son muy poderosos por lo tanto muy respetados, tienen sueños reveladores que muestran el destino de los hombres, sus curas, aparte de lo espiritual y ritual son homeópatas, especialmente cuando usan minerales, alimentos y plantas (Cox, Avelino, 2011).

En el Manual de Salud Comunitaria (URACCAN-IMTRADEC 2012), se menciona que los curanderos forman parte de los Agentes Sanitarios de Salud de la comunidad. Presentan un cúmulo de conocimientos ancestrales, cosmovisión y espiritualidad y atienden enfermedades de origen natural y sobrenatural. Su relación integral con la comunidad es indispensable. Trabajan en forma articulada con el sistema de salud occidental.

\section{Historia oral}

En la era de la comunicación y la sociedad del conocimiento en la que estamos inmersos, la relevancia que tiene el relato de la historia es de vital importancia, especialmente cuando se trata de dar voz a aquellas personas que han sido partícipes de algún momento histórico determinado y que pueden aportar sus visiones acerca de un hecho, suceso o ámbito específico.

El proceso de uso de la historia oral no se reduce únicamente a lo que entendemos por el concepto de "historia", o cuando hacemos uso de lo histórico, es decir, a una serie de acontecimientos sucesivos con fechas y períodos determinados, sino que el uso de la historia oral implica una serie de sucesos, experiencias y, desde luego, sentimientos. Cuando se hace uso de la historia oral se da oportunidad a una persona o colectivo, a contar sus vivencias, experiencias, formas entender y dar significado a su propia vida y así, ayudarnos a comprender la nuestra propia, nuestro presente. A través de nuestro recorrido de indagación pretenderemos dar a conocer el beneficio de la historia oral como material y recurso para analizar lo histórico, así como dar a conocer algunas implicaciones que supone el uso de esta metodología. Además, pretendemos realzar la importancia que tiene el uso del método de recogida de información sobre testimonios orales para dar voz a personas silenciadas.

La historia oral, implica la narración de hechos y sucesos pasados que son expresados a viva voz, con palabras y que, a su vez, permiten que salgan a la luz testimonios de personas desconocidas, "gentes sin historia", fomentando la recuperación de la memoria histórica a través de las vivencias, las experiencias, las prácticas a lo largo de la vida, sensaciones vividas... y que son recogidas de manera escrita (Jiménez, 2009). 


\section{Resiliencia}

En la última década, hemos estado viviendo una gran vorágine de cambios y situaciones que parece que cada vez cuesta más asimilar: crisis económica a escala mundial, revolución tecnológica, nuevo éxodo rural, grandes movimientos migratorios... Los pueblos y los territorios rurales, se convierten además en las zonas con mayor vulnerabilidad ante estas situaciones, sobre todo debido a la realidad geográfica, socioeconómica y demográfica de las mismas, pero hay algo que puede ayudarnos a adaptarnos a ellas e incluso poder sacar algún rédito positivo de las mismas: la resiliencia.

La resiliencia es una capacidad humana de adaptación o superación de circunstancias traumáticas o adversas. Si queremos explicarlo más metafóricamente, podemos imaginarnos una típica pelota antiestrés: podemos aplastarla, estrujarla, estirarla y hacer con ella todo lo que nos venga en gana, pero al final, siempre volverá a su estado original. La resiliencia es precisamente esa capacidad, el poder volver siempre a ser lo que fuimos, o adaptarnos a las realidades más hostiles con las que nos encontramos a lo largo de nuestro camino, permitiéndonos así continuarlo con mayor seguridad y fortaleza. Pero también podemos trabajar la resiliencia a un nivel colectivo y comunitario, de hecho es una buena fórmula de mantener la supervivencia de nuestros pueblos, siendo capaces de enfrentarnos a los problemas que se nos presentan e intentando mantener siempre nuestra esencia.

\section{Cultura}

Cultura no es un objeto entre otros, es un conjunto de relaciones posibles entre ciertos sujetos y su mundo circundante. Está constituida por creencias comunes a una colectividad de hombres y mujeres; valoraciones compartidas por ellos, formas de vida semejantes, comportamientos, costumbres y reglas de conducta parecidos. No son exactamente iguales en todos los individuos, pero presentan rasgos de familia semejantes; son intersubjetivos. Esas disposiciones dan lugar a un mundo propio constituida por una red de objetos (artefactos, obras de consumo o de disfrute) de estructura de relación conforme a reglas (instituciones, rituales, juegos) animado por un sistema significativo común (lengua, mito, formas artísticas)" (Villoro, 1998).

\section{Camino y formas (metodología)}

El proyecto de investigación realizada sobre "Significado de la naturaleza desde la cosmovisión del pueblo rama en las comunidades de Indian River y Punta de Águila del territorio rama - kriol" un factor de fortalecimiento de identidad cultural, siguió los fundamentos de una metodología cualitativa del CRISSAC con enfoque etnográfico, desde la perspectiva del pueblo indígena rama. Esta metodología proporciona los conocimientos para identificar los conocimientos que guardan las personas en la actualidad sobre los significados de la naturaleza hacia el hombre del pueblo rama y 
las experiencias que ha tenido los ancianos y las mujeres indígenas sobre la puesta misma en práctica de los conocimientos ancestrales.

\section{Método de trabajo y estrategia de la investigación}

En la presente investigación se retomaron los principios del CCRISAC (Cultivo y Crianza de Sabidurías y Conocimientos, por ende, se apoyaron de:

- Convivencia con la comunidad indígena rama.

- Conversatorios y dialogo de convivencia sociocultural.

- Diálogos de saberes con ancianos, agricultores y pescadores.

- Vivir la cultura a partir de las prácticas de vida cotidiana, el respeto a la Madre Tierra y la misma naturaleza.

A partir de los conocimientos de los ancianos, adultos de las comunidades se fundamentó en las experiencias de vida con la naturaleza o la Madre Tierra, la predicción sobre el cambio y adaptación climática que afrontan el pueblo indígena rama en su territorio, esto se da mediante la conversación de los ancianos de forma oral (memoria colectiva) entre la interacción del investigador y los participantes claves.

En todo el proceso los actores claves participan de manera directa e indirecta con los pescadores, médico tradicional, partera, agricultores y ancianos de dicha comunidad para la construcción del estudio a través de una comunicación mutua, compartiendo su historia oral, anécdota (mitos y leyendas) sobre la naturaleza.

En el primer momento de conducción para la investigación, se realizó a través de la convivencia con la comunidad y la familia, determinando sus formas de vida entre la naturaleza, que permiten una manera de socialización entre la comunidad.

En el segundo momento se compartió las experiencias y resultados obtenidas, de las historias orales, diálogo de convivencia y las lecciones aprendidas durante y después del estudio y sobre todo la simbología de la naturaleza desde la cosmovisión, a través de la metodología activa participativa que ayuda a construir fundamentalmente el estudio con profundidad, para la identificación de los resultados para la investigación.

\section{Sistematización del CCRISAC/ resultados}

\section{Conocimientos ancestrales en relación a práctica de vida}

\section{Agricultura}

La actividad principal de la Bangkukuk (Punta de Águila) es la agricultura tradicional y sostenible, una práctica ancestral que ha venido conservando y manifestando 
en la familia rama de la comunidad de Bangkukuk Taik. En base a los conocimientos de los agricultores indígenas de Bangkukuk, se afirma que para ellos el cultivo tiene mucho significado en relación al cambio la naturaleza, especialmente en la época de la canícula que entran entre el 15 de julio al 15 de agosto, según los agricultores indígenas, se menciona que dentro de esa fecha no cultivamos la tierra, porque está en un proceso de restauración. A esta razón no es afectivo para los cultivos porque se mueren, por el efecto de lluvias y la poca radiación solar, siendo días que en esa época ocurre con frecuencias la caída de las lluvias, por tanto, perturban las semillas del cultivo y a veces queman las plantas.

La fase lunar otro fenómeno natural que se menciona por los agricultores de la comunidad de Bangkukuk, que en ese período de la luna nueva no se cortan las sepas de plátano, banano, entre otros tubérculos esto lo identifican con la fase lunar. Es recomendable para nosotros como agricultores en el período de la luna llena, cuando la luna nueva todos los seres vivos en la naturaleza esta tiernos y llena sólo de agua el interior, todos los organismos nutritivos no está estable para el crecimiento; mientras, cuando la luna está en la fase llena ya todos los seres en la naturaleza establecen una nutrición con muchas energías vivas, para el nuevo crecimiento y el desarrollo. Todavía hoy en días se conversa la práctica tradicional para cultivar el campo.

En la comunidad de Indian River/ San Juan de Nicaragua es igual la actividad agrícola, lleva la misma explicación de los agricultores indígena que practican los comunitarios basada en una agricultura sostenible y de forma tradicional mediante la simbología de la naturaleza en cuales proporciona a la comunidad al consumo interno de la familia. La única diferencia es la ubicación de la comunidad en el territorio rama.

\section{Vivienda}

Históricamente la vivienda del pueblo rama es de madera y tambo, techo con hojas de palmas una vida de mucha cultura y tradición. En la comunidad de Bangkukuk todavía existen casa de ese estilo, tradicionalmente cuando una familias va a construir su casa se observa el tiempo de la naturaleza, siempre con el apoyo de la luna, es decir cuando la luna esta nueva no se recomienda la construcción de la casa, no es resistente a mas de 5 años, debido que la madera y las hojas de palmas se descomponen en un período corto y muchas veces se agarra comején y hormigas las cuales causan daño a la madera. Mientras si la luna está llena es más resistible para construir una casa indígena porque todos los materiales (madera, poste de palo, hojas de palmas) son durable para un periodo hasta de 15 años duración y resistible.

En Indian River se ha conservado la cultura de mantener su forma tradicional de construir su casa de tambo, siempre haciendo uso de la naturaleza, igual como en Bangkukuk no construye su casa sin ver el calendario, siempre presiden con la fase la luna y la edad de las palmas o árboles para hacer uso de su casa, esto tiene mucha 
relación con la práctica ancestral. Aunque un problema para la obtención de sus materiales hoy en día es que muchos árboles y palmas se han extinguido por fenómenos naturales, el reciente huracán OTTO provocó daños en el 2016, expresa Hilario John (2018).

\section{La caza}

Comparto una historia oral que cuenta respecto al cazador que se dedica mucho a la caza, que no acuerdo mucho del día y el tiempo, pero hay un gusano en la pata del animal, y si usted mata ese animal y obtiene eso, que realmente no es un gusano sino una piedra, eso le trae suerte. La historia cuenta que, un cazador tiene que matar muchos animales para encontrar esa piedra entre las pezuñas, como en el caso del chancho de monte (wari). El que encuentra y saca la piedra nunca más le hace falta la carne. El que la tiene, cada vez que caza un wari tiene que bañar la piedra en la sangre del animal y hay que mantenerla siempre consigo. En el caso del manatí tiene un diamante. El que lo encuentra no debe enseñarlo a nadie porque, es su suerte. Ahora cuando uno tiene esta suerte, muchas veces puede caer en abuso destruyendo más animales de lo necesario, y puede ser castigado por abusivo.

\section{Pesca}

Para la pesca tradicional expresa un pescador que, para la temporada de pesca en el mar, depende del cambio climático que afecta en el momento, pero tradicionalmente como pescador, aprovechamos el tiempo de pesca en el mes de marzo, mayo y septiembre; aunque, puede explicar que todo el año es lo mismo hay muchos cambios en los meses, se observa que existen muchas variaciones de clima. En los meses de marzo, mayo y septiembre sobreviene la pesca de pargo rojo utilizando anzuelo, flecha y arpón. El mes de junio es el mes de la tortuga que tradicionalmente se caza para el consumo de la familia en la comunidad, que por cultura de la comunidad de Bangkukuk Taik, no se vende la carne y se regala a las familias, a modo de vida de la comunidad se ayuda entre la familia y el cazador a limpiar la carne de tortuga para repartir entre todos.

La práctica de la pesca se basa tradicionalmente en un medio de subsistencia, decía un anciano que la pesca en el mar es otro significado diferente que pescar en la laguna; si está amaneciendo con mucho sereno, significa que está amaneciendo con buena temporada para la pesca; otra considera que si la ola del mar no suena mucho eso permite identificar un mayor acceso a la pesca. Si no respeta la naturaleza surgen cambios en el clima, formando olas con viento durante la semana.

El señor Salomón de Indian River argumenta que los indígena ramas, practicaban la pesca tradicional en base a sus creencias, en la pesca ellos usan una sola ropa y la conservan en un lugar que es únicamente para ir a la pesca, la cual considera las formas 
de creencias y la práctica que todavía persisten. Cuando los rama salían de pesca lo hacían por la madrugada antes que salga el sol porque afecta por el resplandor y el rayo solar provoca cambios en el desplazamiento de los peces por la iluminación, y por la tarde cuando se ocultan el sol esto se dan más para la pesca en el río. Otra creencia que expresan es que el pescador tradicional se dirige por medio de la marea, si la marea esta alta no es buena y se sabía por la luna llena y cuando es de luna nueva, los dientes de los peces son suaves y no es bueno para la pesca. Según ellos lo confirman por la calendarización de la fase lunar. Mucho de esto conocimiento ayuda a la nueva generación a contribuir y fortalecer la práctica ancestral.

El señor Cecilio originario de la comunidad de India River, relata que cuando uno va a pescar, hay que tener paciencia, porque el anzuelo es tu suerte, si va a pescar en la noche con arco y flecha es más fácil cazarlos porque ellos están descansando, mientras que en el día los peces están más activos.

\section{Medicina tradicional}

La práctica de la medicina tradicional en la comunidad de Bangkukuk Taik (Punta de Águila) ha sido una ayuda a la familia en especial a los niños(as) que padecen de enfermedades como fiebre, tos u otros. La señora Cristina cuenta que cuando los niños padecen de fiebre, se prepara cilantro, aceite de almendra, un poquito de ron en agua para bañar a los niños y después cubren dentro de una sábana hasta que se quita el calor del cuerpo. El antídoto es una semilla, la hierven para purgante y se toma como cualquier líquido, también dice que es bueno cuando pica una serpiente, no se explica el procedimiento para la preparación simplemente se menciona dentro de la conversación.

En la comunidad de Indian River se formaron un equipo mujeres y hombres que trabajan para su propia comunidad en medicina tradicional, ambos transmiten conocimiento esto significa conservar la identidad cultural del pueblo rama. Este equipo de médicos tradicionales de dicha comunidad ha trabajo parcialmente con el centro de salud al brindar atención a los partos u otras enfermedades como la fiebre amarilla, picadura de serpiente, diabetes, dengue y malaria.

La medicina tradicional representa una simbología de la naturaleza que proporciona medio de subsistencia para la cura de enfermedades, asimismo se menciona un Bush Dakta que es importante conservar y proteger la Madre Tierra, porque sin el bosque o plantas no somos nada; sin embargo, hoy en día están desapareciendo muchas plantas medicinales por algunos efectos naturales y antropológicos. 


\section{La Madre Tierra}

El significado de Madre Tierra, un anciano rama lo traduce en rama kriol Mada Land (Madre Tierra), la riqueza de la tierra desde todo el ecosistema hasta más allá de la cosmovisión que son los conocimientos indígenas con el MOMENT TIME, aunque hay un cambio de la Mada Land hoy en día se está enfermo por muchas afectaciones que causan nosotros como seres humanos que viven en ella. La Mada Land para el pueblo rama manifiesta un ver la tierra como el centro del alma para el ser humano. En una expresión de tristeza por el Bush dacta que la tierra está realizando como revancha a la humanidad por el daño que estamos causando por los malos hábitos, por nuestras actitudes, comportamiento hacia el respeto a la naturaleza donde vivimos.

Si la Madre Tierra proporciona todo a la humanidad la condición apropiada significa que se requiere una de atención del hombre mismo, para valorar su importancia de propiciar las condiciones necesarias para el hombre.

\section{Simbología de la naturaleza indígena basada a la cosmovisión rama}

En la historia oral de miss Cristina, hablante rama manifiesta cuando el mono congo comienza a llorar diario en la mañana, anuncia que pronto comienza a llover fuerte, además se crecen los ríos esto significa una simbología de la naturaleza para el pueblo rama. El reconocimiento para la predicción de tiempo en la ocurrencia de los fenómenos de la naturaleza. Es interesante mencionar que el pueblo rama a través de los animales se predicen los malos tiempos para estar preparados contra la adaptación al cambio de la naturaleza

El señor Ruiz de 36 años de edad, originario de Indian River e indígena rama, comparte uno de los mitos "cuando miramos a las sardinas saltar en los caños viene anuncian que los caños se van a desbordar y cuando miramos que los árboles botan las hojas significa que viene tiempos lluviosos."

Continua diciendo, que tenemos una creencia en el "wanka" conocido como el "wauh" esta es un ave que cuando canta, dice que es muerte, o sea que alguien se va a morir, otro ave es el "picuak" (el bobo chiso) cuando canta el "picuak" está anunciando, al que lo escucha, que va a tener conflicto con otra gente o escuchar buena o mala noticia, igual con el animal que le llamamos "anssbeer" el (oso hormiguero), cuando este animal canta, proclama, sol o lluvia y cuando canta en la tarde cerca del patio significa que se pondrá el sol, los escarabajos y chinches cuando cantan en el monte anuncian sol y lluvia, hay otra que cantan en la tarde, es segura la lluvia.

El kamsik o lucerna es un insecto que se puede apreciar durante la oscuridad, los ancianos explican que cuando el insecto vuela en cantidades encima de los zacates se anuncian muchas lluvias, para ellos se ha comprobado en un 90\% la probabilidad de 
lluvias durante la semana. Esta señal ha demostrado un valor a la naturaleza para el pueblo rama de presidir el tiempo de lluvia. Por lo que considera los ancianos(as) un medio de comunicación entre la naturaleza observar que los insectos son parte del medio natural que nos rodea, se manifiestan señales de lluvias en la zona.

Otra de las creencias es sobre el tigre, la lapa y la tortuga, cada uno tiene una historia que se interrelaciona con la naturaleza, se dice del tigre que cuando lloran anuncian malas noticias o enfermedades, por ejemplo la pérdida de un ser querido, también contacta las emociones, cuando la persona está pensando en la novia, se le aparece como hombre, toma varias figuras y hasta juegan contigo y no te das cuenta, toma voz de niño y animal, en caso de la lapa, cuando cantan en coro anuncian buen tiempo, de lo contrario anuncian lluvia; cuando las tortugas se observan en la rivera de los ríos y caños, predicen que pronto abran inundaciones.

\section{Conclusión}

El trabajo de investigación conducido principalmente en las comunidades de Bangkukuk (Punta de Águila) y Indian River San Juan de Nicaragua, sobre "Significado de la naturaleza desde la cosmovisión del pueblo rama en las comunidades de Indian River y Punta de Águila del territorio rama - kriol" su principal fundamentación basada desde la historia oral y los conocimientos ancestrales del pueblo rama. En este sentido la investigación etnográfica, utiliza la metodología del CRISACC a partir de junio y julio del 2018. La fuente principal y más importante para obtener información sobre el tema fueron los ancianos conocedores de la historia oral de la naturaleza desde la cosmovisión del pueblo rama a través del diálogo del saber y la conversación directa. También fue importante la observación participante en todas las actividades que realizara los/as comunitarios/as en la vida cotidiana durante el tiempo de la investigación etnográfica en las comunidades.

Todas las creencias, la simbología de la naturaleza y las prácticas de los rama están vinculadas con otros seres naturales y sobrenaturales con quienes se comparte el medio ambiente y hábitat principalmente de río, laguna y mar. La mitología y cosmovisión no reconocen o no señala a seres humanos como héroes o seres extraordinarios. Los héroes, los protectores, los que tienen y proporcionan información y conocimiento son los animales que se manifiestan en forma natural y sobrenatural. Esto explica por qué los rama cantan a la naturaleza y a los animales. Se inspiran en la belleza de la naturaleza y en ningún momento en las personas o en personajes.

Es importante la conservación y protección de la naturaleza porque proporcionan fuente de energía hacia el ser humano a través de las plantas para la creación de la medicina natural y medio de subsistencia para la salud. Asimismo, la adaptación al cambio climático desde los conocimientos ancestrales. 
La importancia de los animales en la cosmología rama se manifiesta en las costumbres sencillas de vestimenta, la interacción con otras culturas, todo esto contribuye a una identidad centrada en la naturaleza. Los ramas se reconocen en niveles horizontales de diálogo con los animales, donde sobresale la comunicación con el amigo tigre una comunicación entre su potencialidad de poder para el pueblo rama.

La cosmovisión de los rama constituyen las convivencias o acciones de las creencias e interpretaciones que ellos encontraron mediante las diferentes manifestaciones culturales o experiencias vividas. Estos existen como una tradición oral del pueblo rama a más de muchos años. No existen muchos estudios y recopilación sobre los mitos y leyendas de los ramas. Las creencias no están escritas y es un aspecto a considerar para la enseñanza-aprendizaje entre los mismos del pueblos.

En su cosmovisión se crea un contexto generalizado en el que la gente siente respeto y temor a la naturaleza. La generación mayor tiene más creencia en estos cuentos y son más conservados, pero la generación nueva también se los cree ya que cuando les suceden algunos acontecimientos misteriosos, la mejor explicación son estos cuentos. Por lo menos no se atreven a hacer lo que se considera peligroso. Estas ideas y reglas invisibles, pero incidentes en la mente de los indígenas rama es lo que los ha convertido en mejores cuidadores del bosque.

Los seres humanos creamos cultura. La fuerza de las costumbres y tradiciones no radica en la frecuencia con que la gente las practique, sino en que la misma comparta auténticamente las ideas y creencias que originaron la costumbre y la tradición. En el ejemplo del culto a los muertos, la forma de cultivar la tierra y la misma adaptación al fenómeno natural, el cumplimiento cabal de la tradición depende mucho de que las personas crean de verdad en la existencia de la vida espiritual, en la posibilidad de que los muertos visiten el mundo de los vivos, guiados por la luz para recibir una ofrenda de alimentos, entre otras creencias.

El pueblo rama afirma la importancia de revitalizar los valores culturales, porque así nos ayuda a sentirnos orgullosos de nuestra propia identidad, de tal manera sirve de enseñanza para la nueva generación porque muchos de estos valores están perdiendo cada día, sobre todo promover e implementar una educación intercultural en el territorio. Desde un plan de acción con los comunitarios para promover e implementar un proyecto de vida para la comunidad indígena rama.

\section{Agradecimiento}

Esta publicación obtuvo el financiamiento de: El Fondo de Asistencia Internacional de los Estudiantes y Académicos Noruegos (SAIH). 


\section{Lista de referencia}

Arellano J. E. (2009). La Costa Caribe nicaragüense: desde su origen hasta el siglo XXI. $1^{a}$ ed. Managua. Academia de geografía.

Cuenin, F. (2009). Patrimonio cultural y desarrollo socioeconómico: la recuperación de áreas centrales históricas (Nota Técnica). BID. New York.

Cunningham, M. (2008). Módulo de Espiritualidad, Conocimientos e Historia de los Pueblos Indígenas de Abya Yala. Fondo Indígena, Bolivia.

Cox, A. (2016). Espiritualidad y filosofía indígena. Universidad URACCAN, Nicaragua.

Cox Molina, A. (2011). El vuelo del Alma. Cosmovisión Miskitu. Managua, Nicaragua.

IMTRADEC-URACCAN (2012). Manual de Salud Comunitaria. Managua, Nicaragua

Rivelstone, Gerald. (2008). La tierra de nuestros ancestros territorio rama y creole en el Caribe Nicaragüense. Managua, IBIS.

Rizo Mario (2006). Cultura: Material de apoyo para la formación docente en educación intercultural. Managua, Nicaragua.

Roncal Martínez Federico (2006). La educación y cultura. publicado en Guatemala.

Zamudio Teodora (2002). Conocimiento tradicional del pueblo indígena. $1^{0}$ edición, Ecuador. 